\title{
Experimental study of particle concentration fluctuations in a turbulent steady flow
}

\author{
François-Xavier CIERCO, Mohamed NAAIM, Florence NAAIM-BOUVET \\ Cemagref, Groupement de Grenoble, Unité de Recherche ETNA, 2 rue de la Papeterie, BP 76, \\ 38402 Saint-Martin-d'Hères Cedex, France \\ E-mail: francois-xavier.cierco@ec-lyon.fr
}

\begin{abstract}
Specific features of the physics of blown sand and PVC particles were investigated in windtunnel experiments. The fluctuations in particle concentration over time were derived from image processing of high-speed films $(500 \mathrm{~Hz})$. Spectral analysis of these time series showed a slowly decreasing slope within a frequency range that could be larger than the inertial sub-range of the free stream. A specific transport regime also appears in the lower part of the flow for frequencies less than $10 \mathrm{~Hz}$. The role of turbulence in the generation of this regime is discussed using a simple theoretical model able to describe the aerodynamic behavior of a particle.
\end{abstract}

\section{INTRODUCTION}

The physics of blowing particles, such as sand or snow, in a turbulent boundary layer is governed by different complex processes. When wind speed increases and transport takes place, take-off phenomena are first initiated by aerodynamic entrainment, i.e., particles are picked up by the flow. In the first few centimeters, particles follow ballistic trajectories. Particle movement consists in successive bounds that impact the soil in a process called saltation. However, each impact is also a potential source of ejection. Grains making short hops just above the ground after this ejection are transported by saltation. At higher wind speeds, the particles are transported upward by turbulent eddies and far downwind of the bed. This process is referred to as suspension.

Between these asymptotic behaviors (saltation and diffusion), various changes occur, which is why Foucault and others (1996) proposed four specific behaviors: pure saltation, a modified saltation state, a modified suspension state and pure suspension. These states are characterized by an adimensional number according to the values of timescale ratios and the crossing-trajectory parameter (Tanière and others, 1997).

First the particle relaxation time $\tau_{\mathrm{p}}$ is compared to the timescale $\tau_{\mid}$of the smallest turbulent structures and the timescale $\tau_{\mathrm{L}}$ of the largest turbulent structures. If $\tau_{\mathrm{p}} / \tau_{1} \ll 1$, particles and fluid velocities are similar and the process is pure suspension. If $\tau_{\mathrm{p}} / \tau_{\mathrm{L}} \gg 1$, particles are not influenced by turbulence and the process is pure saltation. In other cases, particle behavior is related to both turbulence and particle inertia effects. But significant effects of particle-wall collisions are expected when saltation is modified, and a substantial effect of turbulence is expected when suspension is modified. The boundary between the two is defined using the ratio between the particle free-fall velocity and the vertical root-mean-square (rms) velocity of the fluid. If this ratio exceeds 1 , the modified saltation state is defined; otherwise, it will be the modified diffusion state.

Because of all these specific features, and the mutual interaction between wind and airborne particles, the physics of wind transport of solid particles is not completely understood.

To gain insight into the interdependence of air and particle movements, the temporal fluctuations of the solid concentration were investigated through experimental measurements, conducted with sand and PVC in a turbulent steady flow obtained in the biphasic wind tunnel at Cemagref, Grenoble, France. Unfortunately, the limited capabilities of the available instrumentation and the high flow velocity required by wind transport $\left(>4 \mathrm{~m} \mathrm{~s}^{-1}\right)$ prevented simultaneous measurements of wind and particle velocity. Therefore, a different solution was chosen: first, anemometry measurements were made in a particle free flow using hotwire probes; second, images of the sand and PVC transport were collected with a fast camera. Image processing and analysis provided an estimation of the particle concentration at different heights and at a high frequency $(500 \mathrm{~Hz})$.

\section{EXPERIMENTAL SETTING}

\subsection{Wind tunnel and measurement technique}

The experimental set-up was specially designed for dynamic studies of aeolian transport (Naaim-Bouvet and Naaim, 1998). The $13 \mathrm{~m}$ long wind tunnel has a $5 \mathrm{~m} \times 1 \mathrm{~m} \times 0.5 \mathrm{~m}$ test area with a uniform cross-section. Wind speed is regulated with a fan, particles are placed on the floor and downstream from the test area where measurements are taken, and particles are collected in the upper part of the wind tunnel with filters that prevent particles from re-entering.

For each experiment, the wind tunnel was run twice at the same wind speed, first without particles, then with loose sand (or PVC) particles. Despite the shortcomings of this procedure, it provides preliminary knowledge. The influence of particles on fluids can be estimated by measuring the fluid velocity in the presence of solid particles, as was done by Tanière and others (1997), who used laser Doppler anemometry to measure the mean and rms velocity of both air and particles. It was shown that the flow was not significantly altered by the particles because of the very low concentration of the solid phase whose local volume, similar to our own experiment, did not exceed $10^{-4}$. More recently, Zhang and others (2007), employing hot-wire anemometry with a robust hot-film probe, showed that the wind profiles with saltating sand particles exhibit a shallower gradient, indicating the momentum extraction by airborne sand particles. This experimental approach was not adopted in the present study because of technical problems associated with measuring the 


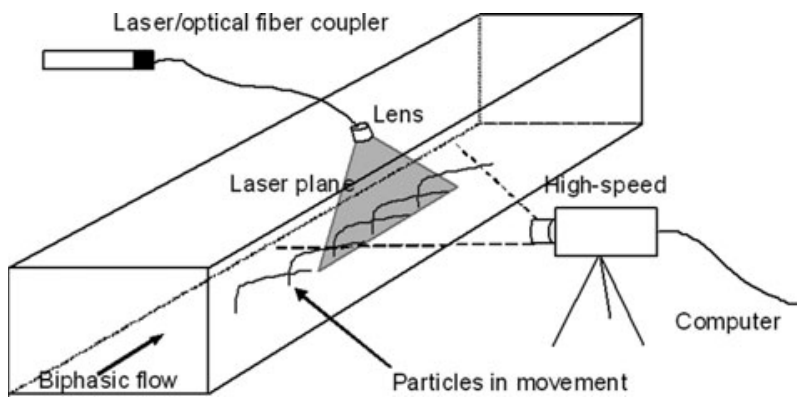

Fig. 1. Experimental setting.

particle-laden flow velocity: laser Doppler anemometry was not available and our hot-wire probe was not sufficiently robust.

Therefore, hot-wire probes were used to collect $1000 \mathrm{~Hz}$ wind velocity measurements during the first run. In the second run, 1600 images were taken at $500 \mathrm{~Hz}$ by a CMOS (complementary metal oxide semiconductor) camera whose resolution $(1024 \times 1280$ pixels $)$ could investigate a $12 \times$ $16 \mathrm{~cm}$ area (each pixel representing a $0.125 \times 0.125 \mathrm{~mm}$ square in the wind tunnel). A laser beam provided the required light sheet (Fig. 1). All measurements were collected in the experimental channel roughly $3.50 \mathrm{~m}$ from the channel entrance and at wind speeds varying from 4.36 to $6.14 \mathrm{~m} \mathrm{~s}^{-1}$.

\subsection{Flow and particle properties}

The boundary layer depth was approximately $10-13 \mathrm{~cm}$ depending on the reference speed. Following White and Mounla (1991), the stream could then be assumed to be undisturbed by the walls and the steady-state conditions were nearly satisfied.

Nine wind profiles were collected in the free flow. The main wind velocity varied from 4.36 to $6.14 \mathrm{~m} \mathrm{~s}^{-1}$ (Fig. 2). Consequently, the Reynolds number was approximately $10^{5}$. These profiles were correctly modeled by the law of the wall on the first 2 or $3 \mathrm{~cm}$ above the ground, which is consistent with the limits of $z^{+}=1000$ (with $z^{+}=z u_{*} / \nu$ ) given by Bailly and Comte-Bellot (2003) for very high Reynolds numbers. The $1000 \mathrm{~Hz}$ frequency used in the measurements was judged sufficient because (1) it allows one to observe the stream's main characteristics and (2) the concentration fluctuations could not be investigated at a rate higher than $500 \mathrm{~Hz}$.

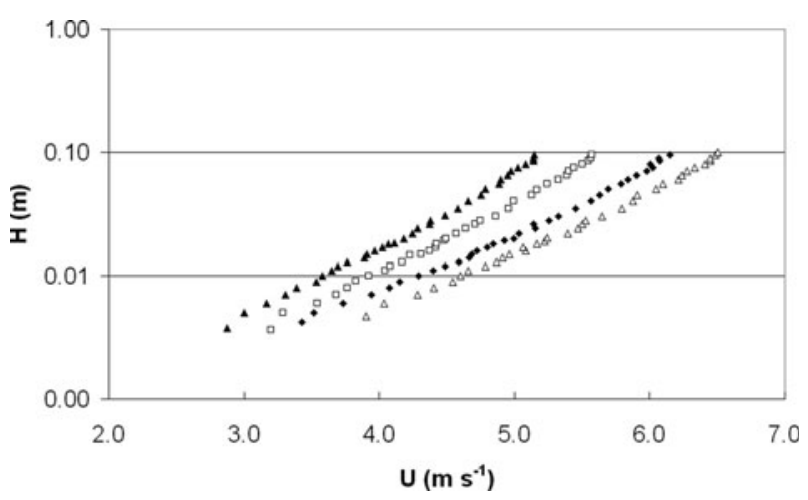

Fig. 2. Logarithmic part of the wind profiles $(z+<1000)$ at different wind velocities.

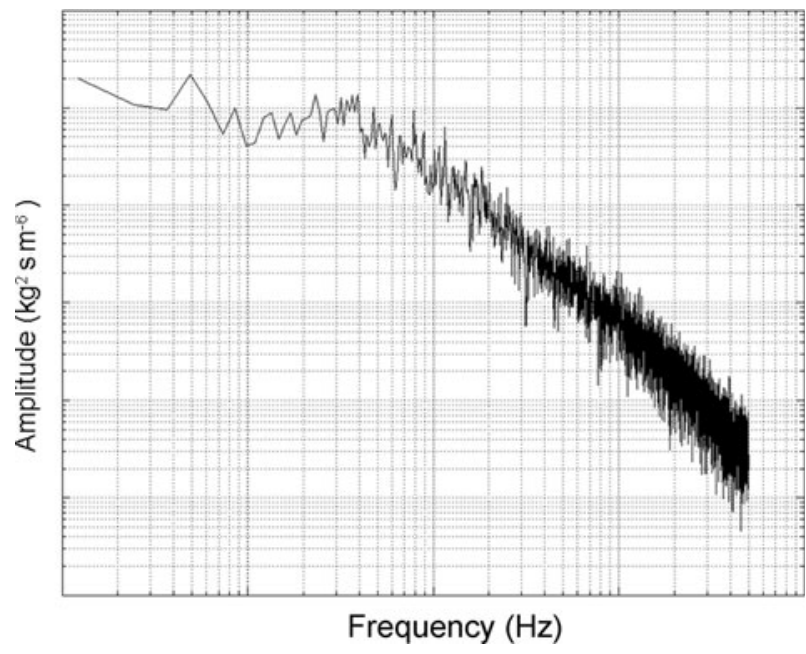

Fig. 3. Longitudinal one-dimensional kinetic energy spectrum computed $5 \mathrm{~cm}$ from the wall at $u_{*}=0.41 \mathrm{~m} \mathrm{~s}^{-1}$.

The kinetic energy spectrum in the $x$ direction was computed at different heights from the wall (e.g. $5 \mathrm{~cm}$ as in Fig. 3). The main wind velocity was $5.54 \mathrm{~m} \mathrm{~s}^{-1}$. The windtunnel size prevents the formation of large turbulent structures whose characteristic scale can be deduced from the $5 \mathrm{~Hz}$ cut-off frequency.

The particle samples were spread evenly over a part of the wind-tunnel section $2.5 \mathrm{~m}$ upwind of the measurement area. In fact, to avoid ripple formation, a short fetch length $(1 \mathrm{~m})$ was selected. This prevented us from obtaining saturation. Nonetheless, the distance traveled by the blown particles between the end of the fetch and the measurement area $(2.50 \mathrm{~m})$ was long enough for the saltation process to reach equilibrium. Two types of particles were used in our experiments: $40-315 \mu \mathrm{m}$ natural sand (mean diameter $108 \mu \mathrm{m})$ and $132 \mu \mathrm{m}$ spherical monodisperse PVC balls.

According to Tanière and others (1997), the timescales $\tau_{\mathrm{l}}$, $\tau_{\mathrm{L}}$ and $\tau_{\mathrm{p}}$ were estimated by

$$
\tau_{\mathrm{l}} \approx\left(\frac{\nu}{(0.1 U)^{3}}\right)^{1 / 2}, \quad \tau_{\mathrm{L}} \approx \frac{\delta}{0.1 U^{\prime}} \quad \tau_{\mathrm{p}}=\frac{U_{\mathrm{F}}}{g},
$$

where $\nu$ is fluid kinematic viscosity $\left(\mathrm{m}^{2} \mathrm{~s}^{-1}\right), U$ is fluid freestream velocity $\left(\mathrm{m} \mathrm{s}^{-1}\right), U_{\mathrm{F}}$ is particle-free velocity $\left(\mathrm{m} \mathrm{s}^{-1}\right)$, $\delta$ is boundary layer thickness $(\mathrm{m})$ and $g$ is gravitational 


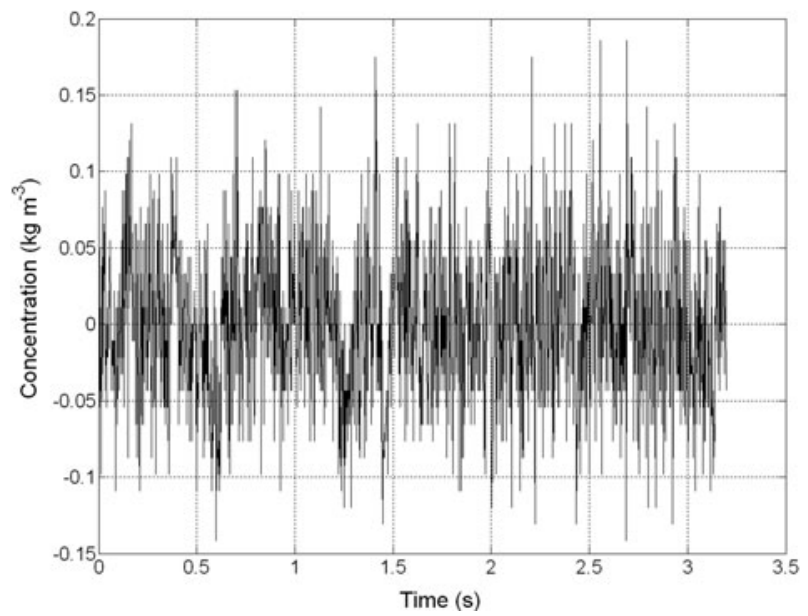

Fig. 5. Sand concentration fluctuations at $u_{*}=0.41 \mathrm{~m} \mathrm{~s}^{-1}$.

acceleration $\left(\mathrm{m} \mathrm{s}^{-2}\right)$. The free-fall velocity is determined using the drag coefficient value proposed by Morsi and Alexander (1972) for a particle Reynolds number ranging between 1 and 10 . The vertical rms velocity of the fluid is estimated by the friction velocity $u_{*}\left(\mathrm{~m} \mathrm{~s}^{-1}\right)$ measured previously. It can be seen from Table 1 that the particles involved are neither in pure suspension nor in pure saltation. A particle-wall interaction and turbulence effects can be expected from this theoretical prediction but must be confirmed by experimental results.

\section{RESULTS}

\subsection{Mean concentration and concentration fluctuation profile}

The concentration profiles were obtained as follows: the collected images were binarized so that the particles were depicted by white areas (each corresponding pixel was then coded 1 ; the others were coded 0 ). Then every image was cut into 256 four-pixel-high strips. The corresponding height $h_{\mathrm{s}}$ of each strip in the experimental channel was $0.5 \mathrm{~mm}$. This strip determines a box whose other dimensions (its length $L$ and depth $d$ ) are given by the experimental conditions $\left(L=L_{x}\right.$ and $d=e$, where $L_{x}$ and $e$ denote the image length and the depth of the laser sheet, respectively). The mass concentration $C_{\mathrm{m}}$ was then derived from the number of particles $N_{p}$ in each box, the box volume, and the

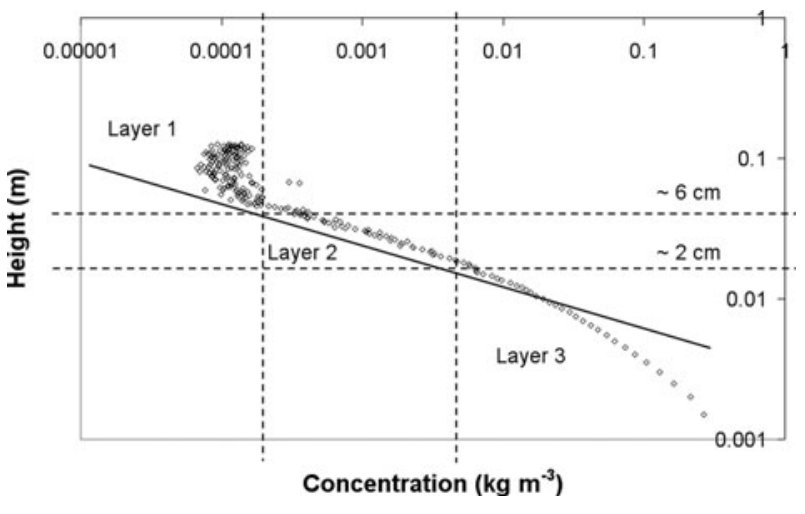

Fig. 6. Sand: mean concentration profile for $u_{*}=0.41 \mathrm{~cm} \mathrm{~s}^{-1}$.

mean diameter $D_{\mathrm{p}}$ and density $\rho_{\mathrm{p}}$ of sand grains:

$$
C_{\mathrm{m}}=\frac{\left(\frac{4}{3} \pi \frac{D_{\mathrm{p}}^{3}}{8}\right) \rho_{\mathrm{p}} N_{\mathrm{p}}}{\left(L_{x} h_{\mathrm{s}} e\right)} .
$$

This can be computed for each of the 1600 images of a film so that the averaged vertical concentration profile and the temporal fluctuations in a given strip could have been obtained (Figs 4 and 5).

After matching the origin of altitudes in the image referential and the wind-tunnel referential, it appears that the concentration peak in the mean concentration profile actually occurs at ground level. This observation indicates that the experimental results are not significant beyond $h_{\mathrm{p}}$ where $h_{\mathrm{p}}$ denotes the peak altitude $\left(h_{\mathrm{p}}=3 \mathrm{~mm}\right)$. The variations in concentration with height for sand and PVC are shown in Figures 6 and 7: the particle concentration decreases rapidly when increasing the height above the particle bed. For both particles, the measures appear to be very noisy in layer 1 because of the very small number of particles per strip. In layer 2, the data can be described by a power law, as previously described by Naaim and Martinez (1995), but in this case the power law fails to describe the mean concentration profile in layer 3 . This abrupt transition at a certain height was also recently shown by Zhang and others (2007), although in this case an exponential law was used.

\subsection{Fluctuation concentration spectra}

\section{Computation}

On each strip, 512 sample blocks were extracted from the initial fluctuation signal and then computed with a fast-
Table 1. Particle characteristics and typical timescale ratio for the present experiments (considering $\delta=0.13 \mathrm{~m}, U=6.5 \mathrm{~m} \mathrm{~s}^{-1}$ $u_{*}=0.41 \mathrm{~m} \mathrm{~s}^{-1}$ )

\begin{tabular}{lcc}
\hline & Sand & PVC \\
\hline Particle density $\rho_{\mathrm{p}}\left(\mathrm{kg} \mathrm{m}^{-3}\right)$ & 2650 & 1450 \\
Mean diameter $d_{\mathrm{p}}(\mu \mathrm{m})$ & 108 & 132 \\
$U_{\mathrm{F}}\left(\mathrm{m} \mathrm{s}^{-1}\right)$ & 0.52 & 0.66 \\
$\tau_{\mathrm{p}}$ & 0.053 & 0.067 \\
$\tau_{\mathrm{p}} / \tau_{\mathrm{I}}$ & 19.88 & 25.14 \\
$\tau_{\mathrm{p}} / \tau_{\mathrm{L}}$ & 0.265 & 0.335 \\
$U_{\mathrm{F}} / U_{*}$ & 1.27 & 1.6 \\
& & \\
\hline
\end{tabular}

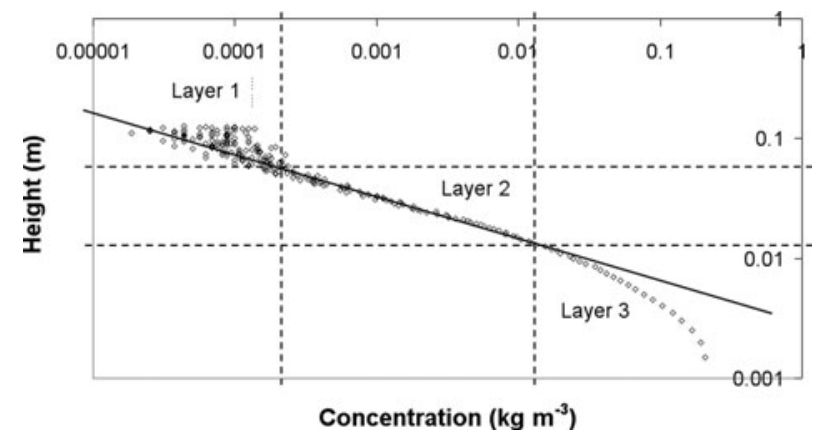

Fig. 7. PVC balls: mean concentration profile for $u_{*}=0.41 \mathrm{~cm} \mathrm{~s}^{-1}$. 


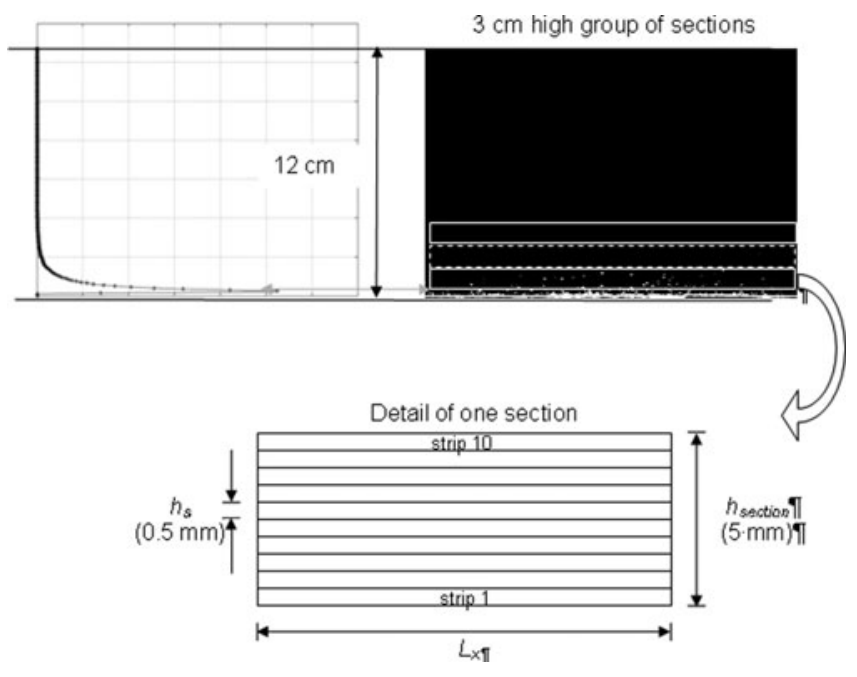

Fig. 8. Organization of strips and sections in the overall image.

Fourier-transform algorithm. Finally, five initial spectra were obtained for every strip of each film.

On each image, the area that matched the first $3 \mathrm{~cm}$ above $h_{\mathrm{p}}$ was also cut into six $5 \mathrm{~mm}$ high sections (each section containing exactly ten strips as shown in Fig. 8). Each section was attributed a spectrum that resulted from the average of the corresponding initial spectra, i.e. the five spectra of all ten strips contained in the section.

The available spectral range was determined by the sampling rate $f$ and the total number of samples $N_{s}$. Because of the Shannon criterion, the spectral range is limited to $\left[f / N_{s}\right.$ $f / 2$ ]. Here $f=500 \mathrm{~Hz}$ and $N_{\mathrm{s}}=512$ so that the $1-250 \mathrm{~Hz}$ sub-range can be studied.

\section{Spectra analysis}

Higher than $1 \mathrm{~cm}$ above $h_{\mathrm{p}}$, the fluctuation concentration spectrum can be described by a single slowly decreasing trend whose $-1 / 5$ slope is evidence that the particle concentration does not behave like a passive scalar (Fig. 9).

Close to the wall, the spectra of the particle concentration fluctuations clearly appear to be split into two parts from either side of a specific frequency, as depicted in Figure 10. A sharply decreasing slope (approximately -1) appears in the $1-10 \mathrm{~Hz}$ range of the concentration fluctuation spectrum, whereas the slope is rather small (approximately -1/5) at higher frequencies $(10-100 \mathrm{~Hz})$. The magnitude of the

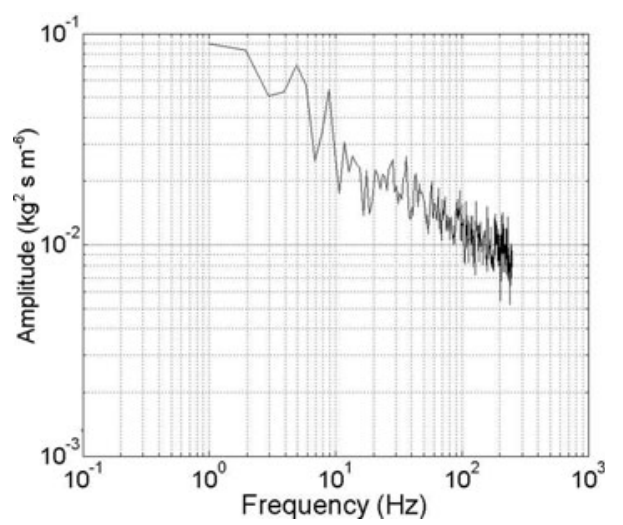

Fig. 9. Fluctuation spectrum (wind speed $5.99 \mathrm{~m} \mathrm{~s}^{-1}$ ). Height 25$30 \mathrm{~mm}$; length $16 \mathrm{~cm}$.

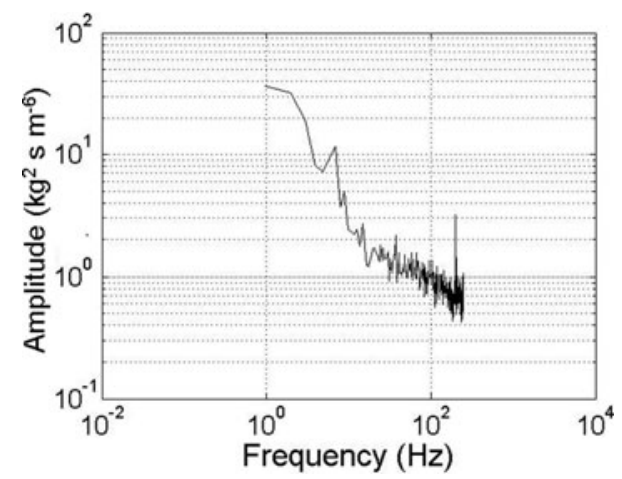

Fig. 10. Fluctuation spectrum (wind speed $5.99 \mathrm{~m} \mathrm{~s}^{-1}$ ). Height above the ground $0-5 \mathrm{~mm}$; length $16 \mathrm{~cm}$.

characteristic frequency $f_{\mathrm{C}}$ that splits the spectrum at $0.5 \mathrm{~cm}$ is $10 \mathrm{~Hz}$. The main wind velocity ranged from 4.36 to $6.14 \mathrm{~m} \mathrm{~s}^{-1}$. Thus, the wind velocity at $0.5 \mathrm{~cm}$ can be extrapolated from Figure 2 to range between 2.5 and $4 \mathrm{~m} \mathrm{~s}^{-1}$. A cutoff length $L_{C}$ can then be associated with the characteristic frequency $f_{\mathrm{C}}$ :

$$
L_{\mathrm{c}}=U(z) / f_{\mathrm{c}} .
$$

The magnitude of $L_{\mathrm{c}}$ is $24-40 \mathrm{~cm}$, i.e. $1.5-2$ times greater than the image length $L_{x}$. Since the wind speed at $0.5 \mathrm{~cm}$ has been provided by experimental results collected in a free flow, wind speed could have been overestimated, indicating that $L_{c}$ and $L_{x}$ could be of comparable magnitude. This statement could mean that the observation of the cut-off frequency $f_{\mathrm{c}}$ may be a consequence of the data processing. To verify this, the process was run again after each image was reduced to its vertical $2 \mathrm{~cm}$ wide central strip (the image length $L_{x}$ was then reduced to $2 \mathrm{~cm}$ ). The two regimes appear whatever the length of the image, and $f_{\mathrm{C}}$ was plotted against $U(z)$ for every experiment (see Fig. 11). The cut-off frequency appears to be more dependent on the wind velocity than on the image length. It is clear that these two regimes do not result from data processing and therefore only physical causes can explain what occurred close to the wall. The two main processes that could be reasonably involved in concentration fluctuations seem to be fluid turbulence and the random processes of new saltatingparticle ejection at the wall. The following focuses exclusively on fluid turbulence effects.

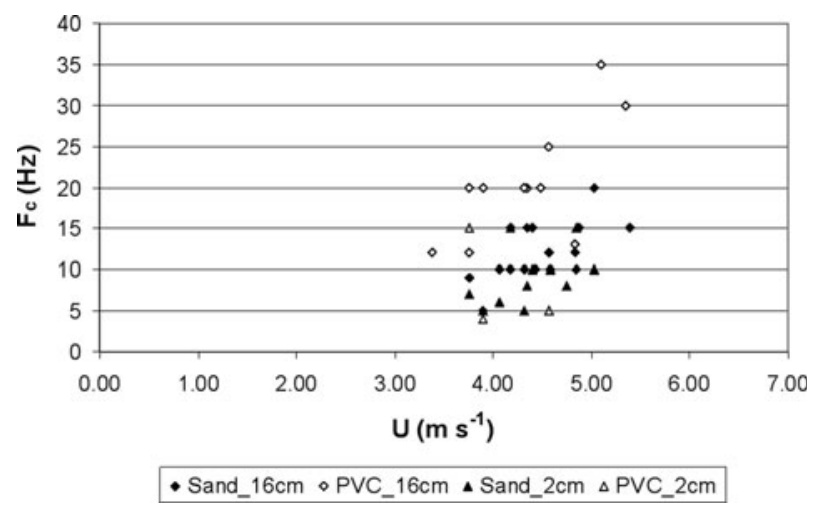

Fig. 11. Influence of wind velocity $U$ and image length $L_{x}$ on the characteristic frequency $f_{\mathrm{c}}$. 


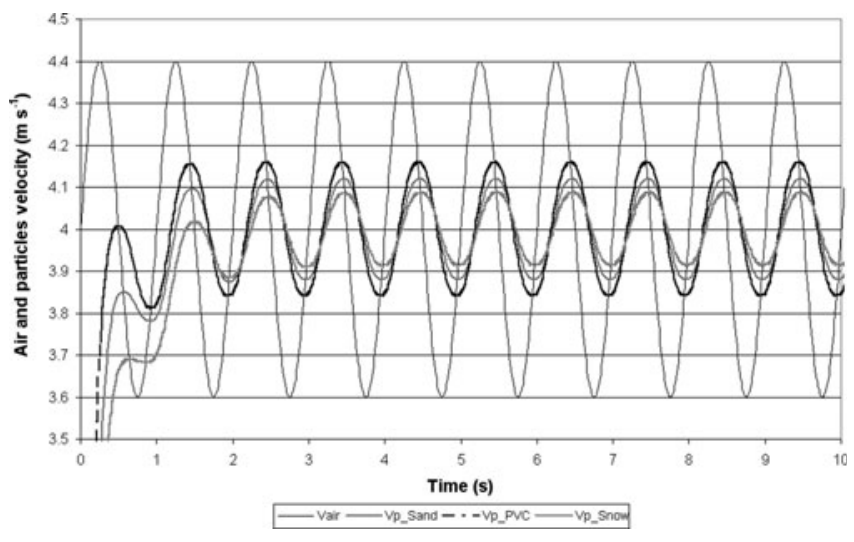

Fig. 12. Particle velocity vs time.

\section{DISCUSSION}

Let us imagine a particle of density $\rho_{\mathrm{p}}$ with initial velocity 0 in a sinusoidal flow. First, the particle velocity increases because of drag forces, which can be modeled by:

$$
\frac{\pi D_{\mathrm{p}} \rho_{\mathrm{p}}}{6} \frac{\partial \overrightarrow{v_{\mathrm{p}}}}{\partial t}=\frac{1}{2} \rho_{\mathrm{a}} S C_{x}\left|v_{\mathrm{a}}-v_{\mathrm{p}}\right| \overrightarrow{\left(v_{\mathrm{a}}-v_{\mathrm{p}}\right)},
$$

where $\rho_{\mathrm{p}}$ and $\rho_{\mathrm{a}}$ denote the particle and air density, respectively, whereas $v_{p}$ and $v_{a}$ represent the absolute particle velocity and the fluid velocity, respectively. $v_{a}$ was assumed to have sinusoidal fluctuations of a given amplitude $A_{\mathrm{i}}$ and frequency $f_{\mathrm{i}}$ around a mean value $v_{\text {mean }}$ :

$$
v_{\mathrm{a}}=v_{\text {mean }}\left[1+A_{\mathrm{i}} \sin \left(f_{\mathrm{i}} t\right)\right] \text {. }
$$

$v_{\mathrm{p}}$ was computed numerically with Equation (3). The computation was interrupted after 1000 iterations, and $v_{\mathrm{p}}$ appeared to be a new sinusoid of lower amplitude $A_{\mathrm{p}}$ and identical frequency $f_{i}$ (see Fig. 12).

Figure 13 shows how the $A_{\mathrm{p}} / A_{\mathrm{i}}$ ratio evolves with respect to $f_{\mathrm{i}}$ for three different types of particles (PVC $132 \mu \mathrm{m}$, $1410 \mathrm{~kg} \mathrm{~m}^{-3}$; sand $108 \mu \mathrm{m}, 2650 \mathrm{~kg} \mathrm{~m}^{-3}$; and snow $300 \mu \mathrm{m}$, $900 \mathrm{~kg} \mathrm{~m}^{-3}$ ). The fluctuations of the particle velocity are largely dependent on the solicitation frequency. The amplitude of the fluctuations is reduced by more than $80 \%$ if their initial frequency is more than $10 \mathrm{~Hz}$. This particular frequency can be compared to what splits the concentration spectrum. Moreover, the velocity attenuation also appears to be governed by two different regimes, as shown in Figures 13

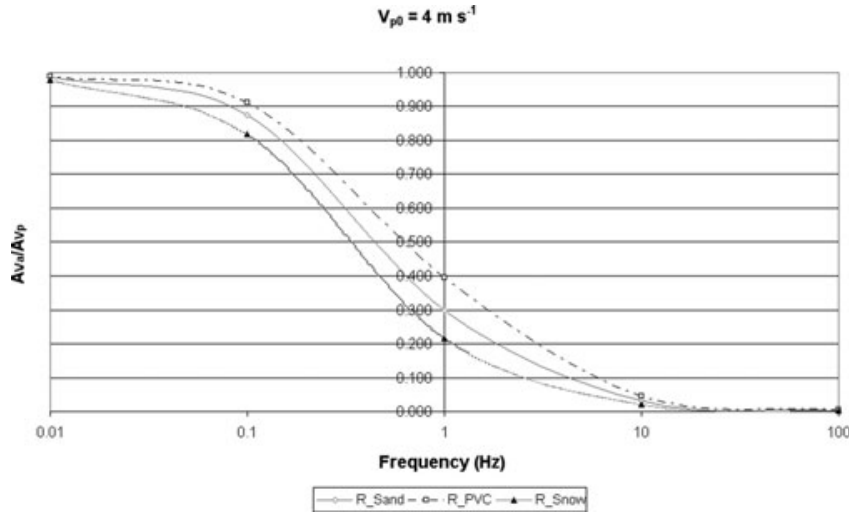

Fig. 13. Attenuation of the wind fluctuations in the simulated particle velocity.

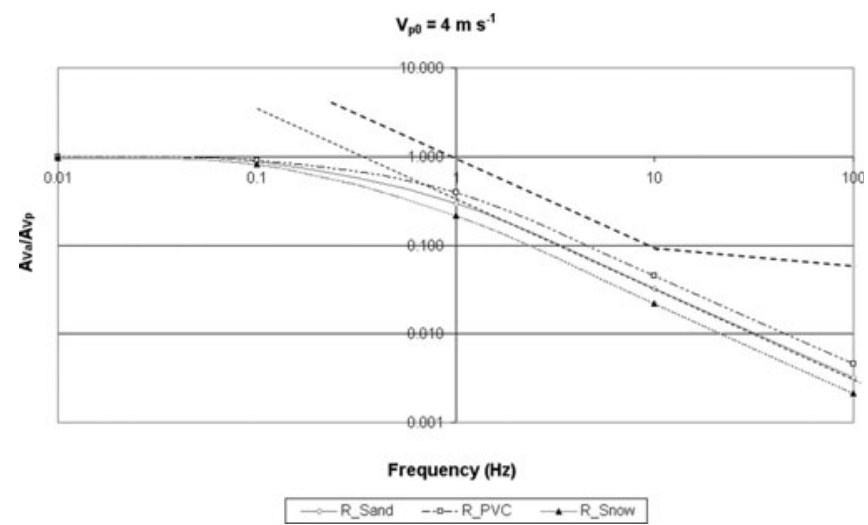

Fig. 14. Log-log representation of Figure 13. The shape of the fluctuation concentration spectrum appears in green (dotted line).

and 14. Nevertheless, the magnitude of the transition frequency is less than $1 \mathrm{~Hz}$, which is quite different from the values observed for $f_{\mathrm{c} \text {. }}$. Last, this model is completely independent of $z$, and wind velocity plays a very minor role in the results presented in Figure 13. Thus, it seems very difficult to find consistent explanations for the appearance of a new regime of concentration fluctuations stemming only from this simple model. More extensive work is required to clarify this point.

\section{CONCLUSIONS}

Filmed sequences of blown sand and PVC particles in a steady flow were obtained at a high frequency $(500 \mathrm{~Hz})$, and the concentration fluctuation spectra were derived from these films. In the vicinity of the ground, two distinct regimes were observed, corresponding to two spectral subranges delimited by a specific frequency. The concentration fluctuation spectrum is therefore characterized by a steep slope within the $1-10 \mathrm{~Hz}$ sub-range even though the slope remains unchanged for high frequencies. Many physical processes can be involved in the appearance of this specific low-frequency regime, among which turbulence and random saltation processes seem the most reasonable. The influence of the fluid-dynamics effects was studied using a simple model. Simulations from this model show that the amplitude of the particle fluctuations is lower than 10-20\% of the amplitude of the fluid velocity fluctuations if the frequency is higher than $10 \mathrm{~Hz}$. Interestingly, this value is the same magnitude as the cut-off frequency in the concentration fluctuation spectrum. Therefore, turbulence plays an important role in the behavior of the particles involved, even close to the wall, as suggested by Tanière and others (1997). Additional research is needed to explain the vertical distribution of the cut-off frequencies observed in the experimental data that are not reproduced by the model and to study the influence of the rebounds and ejections on the concentration fluctuations.

\section{REFERENCES}

Bailly, C. and G. Comte-Bellot. 2003. Turbulence. Paris, CNRS Editions.

Foucault, J.-M., A. Tanière and M. Stanislas. 1996. A criterion for particle suspension in a turbulent boundary layer. C. R. Acad. Sci. [Paris], 322(4), 291-294. 
Morsi, S.A. and A.J. Alexander. 1972. An investigation of particle trajectories in two-phase flow systems. J. Fluid Mech., 55(2), 193-208.

Naaim, M. and H. Martinez. 1995. Experimental and theoretical determination of concentration profiles and influence of particle characteristics in blowing snow. Surv. Geophys., 16(5-6), 695-710.

Naaim-Bouvet, F. and M. Naaim. 1998. Snowdrift modelling in a wind tunnel: vertical and horizontal variation of the snow flux. Ann. Glaciol., 26, 212-216.

Tanière, A., B. Oesterlé and J.C. Monnier. 1997. On the behaviour of solid particles in a horizontal boundary layer with turbulence and saltation effects. Exp. Fluids, 23(6), 463-471.

White, B.R. and H. Mounla. 1991. An experimental study of Froude number effect on wind-tunnel saltation. Acta Mech., Suppl. 1, 145-157.

White, B.R. and J.C. Schulz. 1977. Magnus effect in saltation. J. Fluid Mech., 81(3), 497-512.

Zhang, W., Y. Wang and S.J. Lee. 2007. Two-phase measurements of wind and saltating sand in an atmospheric boundary layer. Geomorphology, 88(1-2), 109-119. 